\title{
Seroconversion after Recombinant Hepatitis B Vaccination
}

\author{
ABHIJIT GUHO, ${ }^{1}$ MD. ABDULAHAD, ${ }^{2}$ MD. ABDUS SALAM, ${ }^{3}$ MD. ABDUL ALIM, ${ }^{4}$ AKM ENAMUL HAQUE, ${ }^{5}$ QUAZ \\ TARI KULISLAM ${ }^{6}$
}

\begin{abstract}
Background: Hepatitis B virus infection is an important public health problem with significant morbidity and mortality. Recombinant hepatitis $B$ vaccine for the prevention of hepatitis $B$ virus infection is in practice in different parts of the world since its availability in 1986. Government of Bangladesh has also included hepatitis B vaccine in EPI schedule since 2005.

Materials and methods: This study was carried out to assess the seroconversion status among hepatitis $B$ vaccinated individuals. A total of 190 individuals including 150 vaccinated persons and 40 non-vaccinated apparently healthy individuals were included as study population. Sources of vaccinated persons were from both EPI and nonEPI schedule of vaccination. Age and sex matched non-vaccinated individuals served as controls for the study. All individuals constituting the study population were screened for HBsAg by Immunochromatographic strip test and only HBsAg-negative persons were included for estimation of their anti-HBs titer.

Results: Out of I 50 vaccinated individuals, 133(88.67\%) were found to have anti-HBs titer in the protective level (> I 0 IU/L), while I 7(I I.33\%) individuals had anti-HBs titer below the protective level (<10 IU/L). All non-vaccinated controls had anti-HBs titers below the protective level. Regarding immune response developed among vaccinated individuals, $67.78 \%, 23.33 \%$ and $8.89 \%$ were good-responders, hypo-responders and non-responders respectively. Mean titer of anti-HBs was found significantly higher among recipients who received booster dose than those who received 3 doses schedule (863.39 IU/L vs. $262.40 \mathrm{IU} / \mathrm{L}$ ), indicating high antibody titer develops after booster dose. Vaccinated group included 85 (56.67\%) men and 65 (43.33\%) women with protective level of anti-HBs titer found in $85.88 \%$ male and $92.31 \%$ female individuals. There was no significant difference of anti-HBs titer between male and female ( $p>0.05)$. Vaccinated individuals from lower socioeconomic condition have had comparatively low rate of protective antibody than people from middle and upper classes.
\end{abstract}

Conclusion: Recombinant HB vaccine induces good level of protective immunity among vaccinated persons.

Keyword: Hepatitis B, hepatitis B vaccine, seroconversion, Bangladesh

\section{Introduction}

Viral hepatitis refers to the inflammation of the liver caused by viral agents. Of the hepatotrophic viruses, hepatitis B virus (HBV) is the most important for its significant morbidity and mortality among infected persons. Hepatitis B virus infection is one of the most prevalent public health problems worldwide. It is estimated that one third of world population have already been infected with this virus. ${ }^{1}$ Globally there are more than 350 million carriers of hepatitis $B$ virus and 0.5 to 1.2 million people die of hepatitis B infection annually. ${ }^{2,3}$ The prevalence of HBV infection varies markedly among different geographical regions and chronic HBV infection can be categorized as high, intermediate and low endemicity.
The age at the time of infection is associated with the endemicity. ${ }^{4}$ In highly endemic areas (carriage rate $8 \%$ or more), infection in infancy and childhood accounts for most of the chronic carriage, whereas in moderately endemic areas (carriage rate 2-7\%), significant number of infections occur in adolescents and adults. ${ }^{5,6}$ Most developed countries fall under low endemic areas (carrier rate $0.5-2 \%$ ) and infections mostly found among the adolescents and adults of relatively well defined high risk groups including injectable drug users, homosexuals, health care workers, patients receiving frequent blood transfusions and or on haemodialysis. ${ }^{7}$

In Bangladesh, information about the prevalence of hepatitis $\mathrm{B}$ infection is scarce and a few sporadic data are available.

1. Lecturer, Department of Microbiology, Khulna Medical College, Bangladesh.

2. Associate Professor, Department of Gastroenterology, Khulna Medical College, Bangladesh.

3. Assistant Professor, Department of Microbiology, Rajshahi Medical College, Bangladesh.

4. Associate Professor, Department of Gastroenterology, Rajshahi Medical College, Bangladesh.

5. Lecturer, Department of Community Medicine, Rajshahi Medical College, Bangladesh.

6. Professor, Department of Medicine, Dhaka Medical College, Bangladesh.

Correspondence: Md. Abdul Ahad, Associate Professor, Department of Gastroenterology, Khulna Medical College, Bangladesh. 
Seroprevalence surveys among men and women in both Bangladesh and India were found to be similar in facts and figures in terms of the rate of exposure and chronic carriage. ${ }^{8}$ In a community-based surveys carried out in Bangladesh, the carriage rate was found to be $5.7 \%$ and $9 \%$ among urban and rural male population respectively and slightly lower rates were reported among women in the same locations. ${ }^{9}$ Another study conducted in rural Bangladesh showed that overall seroprevalence of HBsAg was 6.4\%. ${ }^{10}$ Around 3.5\% of the pregnant mothers in Bangladesh are reported to be carriers of HBV. ${ }^{11}$ Further, 33.3\% patients of hepatocellular carcinoma were found HBsAg positive in their sera in Bangladesh. ${ }^{12}$

HBV spreads through contact with infected body fluids and human is the only natural host. Blood is the most important vehicle for its transmission but other body fluids including semen and saliva have also been implicated. ${ }^{13,14}$ Currently, three modes of HBV transmission have been recognized: perinatal, sexual and parenteral transmission. There is no reliable evidence that airborne infections occur and feces are not a source of infection. HBV is not transmitted by contaminated food or water, insects or other vectors.

Two types of vaccine are currently available, one is plasma derived and another is recombinant hepatitis $B$ vaccine. The inactive plasma derived first generation hepatitis $B$ vaccine became available in 1982. The second generation of hepatitis $B$ vaccine, a DNA recombinant $H B$ vaccine was available for general use in 1986. Both of the vaccines were proven to be safe and efficacious in preventing HBV infection. The most licensed recombinant DNA hepatitis vaccine is a product of $S$ gene consists of 226 amino acids. The recombinant vaccine most widely used is produced in yeast by inserting $S$ gene in plasmid downstream to three genes of Saccharomyces cerevisiae, yeast that serves to promote production of HBsAg. The protein HBsAg is then purified by several physical techniques.

The efficacy of recombinant vaccine is claimed to be more than 95\% among children and 90\% among normal healthy individual. ${ }^{15}$ In 1991, the World Health Organization recommended that hepatitis $B$ vaccination should be included in national immunization program for all countries with a hepatitis B carrier prevalence of 8\% or greater by 1995 and in all other countries by 1997.

The immunity of HB vaccination is directly related to the development of anti-HBs antibodies with a minimum level of $10 \mathrm{IU} / \mathrm{L}$ is considered as protective immunity. ${ }^{3}$ Most people develop antibody titer $>100$ IU/L within 6-8 weeks after completing vaccination. ${ }^{16}$ Some apparently healthy individual do not show an anti-HBs antibody response or respond poorly to the surface antigen component (HBsAg ) and they are labeled as non-responders or hypo-responder with antibody titer $<10$ IU/L and 10-100 IU/L respectively. ${ }^{17}$ It is estimated that about $5-15 \%$ of the vaccinees may be non responder. ${ }^{18}$ However dose, storage, site and route of administration, male sex, genetic factor, obesity, diabetes, and immunosuppression can all adversely affect the immune response.

Post vaccination testing for antibody titer within 1 to 6 months after completion of vaccination series is recommended to detect non-responder or hypo-responder. ${ }^{19}$ Although antibody titer declines with time but it should be reasonably $>10 \mathrm{IU} / \mathrm{L}$ at any time for ensuring immunoprotection among vaccinated individuals. ${ }^{3}$ Although the practice of vaccination against HBV has been started in our country for quite a few years but up till now there is no published data regarding the immunity level among the recipients of HB vaccines of different commercial brands available. This present study has been undertaken to evaluate the status of seroconversion by measuring anti-HBs antibody titer among the recipients of recombinant hepatitis $\mathrm{B}$ vaccine among both EPI and non-EPI vaccinated individuals after completion of their vaccine schedule.

\section{Materials ad Methods}

This was a case control study where study cases included 150 recipients of different age and sex of recombinant $\mathrm{HB}$ vaccine who completed their vaccination either in Expanded Program on immunization (EPI) or non-EPI schedule. The study cases were divided into 5 groups designated as A, B, C, D and E. Each group included 30 cases. Groups are as follows.

Group A: EPI vaccinated children within 1 to 6 months of their $3^{\text {rd }}$ dose.

Group B: EPI vaccinated children after 6 months of their $3^{\text {rd }}$ dose.

Group C: Vaccinated adults after 1 month of their $3^{\text {rd }}$ dose.

Group D: Vaccinated adults after 1 month of their booster dose.

Group E: Vaccinated adults after 6 months of their $3^{\text {rd }}$ and booster dose.

Forty apparently healthy non-vaccinated individuals of age and sex match were included as control. Written consent was taken from each case and control or from their legal guardians before venipuncture for blood collection. The persons who were negative for HBsAg screening test were selected as study population. Blood were aseptically 
collected from both cases and controls. A single sample of $5 \mathrm{ml}$ venous blood was collected using disposable syringe and needle from both cases and control after disinfecting the selected venipuncture site with $70 \%$ alcohol. Collected blood samples were taken in sterile test tubes and labeled with patient's identification number and date. It was kept upright for half an hour and then centrifuged at $3000 \mathrm{rpm}$ for 10 minutes. After centrifugation, the supernatant serum was pipetted into a labeled eppendorf tube with sterile pipette. All samples were stored at $-20^{\circ} \mathrm{C}$ and transported to the Department of Virology, BSMMU, Dhaka in an ice box. Sera from 150 recipients of recombinant $\mathrm{HB}$ vaccine of different age and sex who completed their vaccine either in EPI or non-EPI schedule and 40 apparently healthy non-vaccinated individuals were tested for anti-HBs antibody titer.

The lowest protective value was considered as $10 \mathrm{IU} / \mathrm{L}$ according to the WHO reference value. Therefore, the kit cut-off value was defined as $10 \mathrm{IU} / \mathrm{L}$. So the test sample with an antibody titer below 10 IU/L was considered as nonresponder to hepatitis B vaccination. Antibody titer between 10-100 IU/L was considered as hypo-responder and antibody titer above 100 IU/L was taken as good-responder. Chi square test was used for analysis of data. Mean titer was calculated by Microsoft Excel software.
All relevant information of the study population was recorded in a pre-designed data sheet. The study was carried out from July, 2007 to June, 2008. Laboratory works were performed in the department of Microbiology of Rajshahi Medical College, Rajshahi and Virology department of Bangabandhu Seikh Mujib Medical University (BSMMU), Dhaka, Bangladesh.

\section{Results}

Table-I shows the results of anti-HBs titer among the vaccinated and non-vaccinated individuals. Out of 150 vaccinated individuals, 133(88.67\%) had their anti-HBs titer in the protective level (>10 IU/L), while 17(11.33\%) had antiHBs titer below the protective level ( $<10 \mathrm{IU} / \mathrm{L})$. All nonvaccinated individuals (100\%) were found to have the antiHBs titer $<10 \mathrm{IU} / \mathrm{L}$.

Table-II shows the anti-HBs response among the vaccinees in terms of good-responder, hypo-responder and nonresponder within 1 to 6 months of their vaccination schedule. Out of 90 vaccinees, 8(8.89\%) were non-responders (antiHBs level <10 IU/L), 21(23.33\%) were hypo-responders (antiHBs level between 10-100 IU/L) and 61(67.78\%) were goodresponders (anti-HBs level >100 IU/L).

\section{Table-I}

Results of anti-HBs titer among vaccinated and non-vaccinated individuals

\begin{tabular}{|c|c|c|c|}
\hline \multirow[t]{2}{*}{ Study population } & \multicolumn{2}{|c|}{ Anti-HBs titer $(\mathrm{IU} / \mathrm{L})$} & \multirow[t]{2}{*}{ Total } \\
\hline & Protective $(\geq 10 \mathrm{IU} / \mathrm{L})$ & Non-Protective $(<10 \mathrm{IU} / \mathrm{L})$ & \\
\hline Vaccinated & $133(88.67 \%)$ & $17(11.33 \%)$ & 150 \\
\hline Non-vaccinated & $0(00.00 \%)$ & $40(100.00 \%)$ & 40 \\
\hline Total & 133 & 57 & 190 \\
\hline
\end{tabular}

Table-II

Distribution of good, hypo- and non-responders among the vaccinees

\begin{tabular}{lccc}
\hline Participants & \multicolumn{2}{c}{ Anti-HBs titer (IU/L) } \\
\cline { 2 - 4 } & Non-responder & \multicolumn{2}{c}{ Responder } \\
\cline { 3 - 4 } & $(<10 \mathrm{IU} / \mathrm{L})$ & $\begin{array}{c}\text { Hypo-responder } \\
(10-100 \mathrm{IU} / \mathrm{L})\end{array}$ & $\begin{array}{c}\text { Good-responder } \\
(>100 \mathrm{IU} / \mathrm{L})\end{array}$ \\
\hline Group A $(\mathrm{n}=30)$ & $05(16.67 \%)$ & $15(20.00 \%)$ & $13(43.33 \%)$ \\
Group C $(\mathrm{n}=30)$ & $02(6.67 \%)$ & $00(00.00 \%)$ & $29(96.67 \%)$ \\
Group D $(\mathrm{n}=30)$ & $01(3.33 \%)$ & $21(23.33 \%)$ & $61(67.78 \%)$ \\
\hline Total & $8(8.89 \%)$ & &
\end{tabular}


Figure-1 shows anti-HBs titer among the vaccinated individuals. Out of 150 vaccinated individuals, anti-HBs titer in 17 (11.33\%) individuals were with less than $10 \mathrm{U} / \mathrm{L}, 34$ (22.67\%) had titer between 10-99 IU/L, 83 (55.33\%) had titer between 100-1000 IU/L and 16 (10.67\%) individuals had the titer over $1000 \mathrm{IU} / \mathrm{L}$.

Table-III shows the comparison of anti-HBs titer between $3^{\text {rd }}$ and booster dose of vaccine among the adults. A total number of 30 adults who received $3^{\text {rd }}$ dose of hepatitis $B$ vaccine (0, 1 and 2 months), mean titer of anti-HBs were $262.40 \mathrm{IU} / \mathrm{L}$ after 1 month of completion of their $3^{\text {rd }}$ dose. Among the 30 individuals, who received booster dose including 3 doses, mean titer of anti-HBs were 863.39 IU/L after 1 month of their booster dose. Mean titer was found significantly higher in booster dose than in $3^{\text {rd }}$ dose.

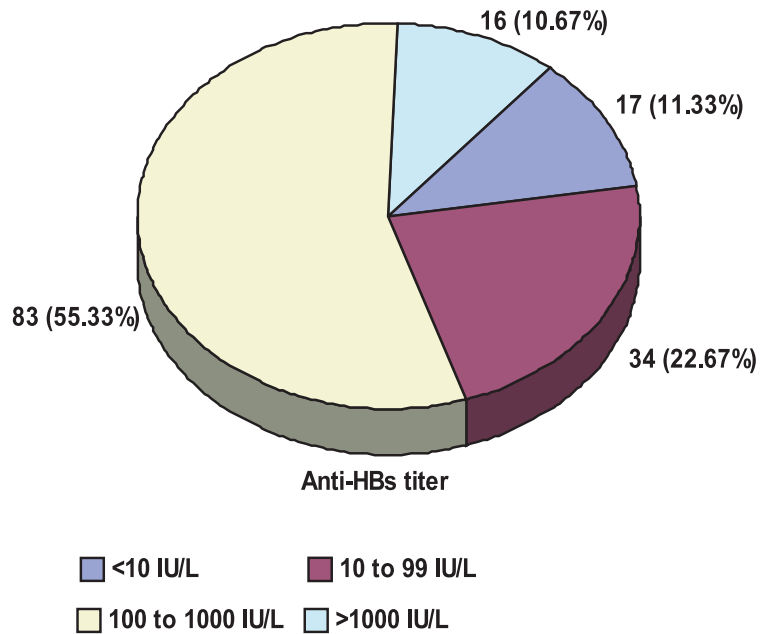

Fig.-1: Anti-HBs response among the vaccinated individuals $(n=150)$

Table-III

Comparison of anti-HBs titer between recipients of $3^{\text {rd }}$ dose and booster dose of vaccine among the adults

\begin{tabular}{cc}
\hline 1 month after $3^{\text {rd }}$ dose $(\mathrm{n}=30)$ & Anti-HBs titer $(\mathrm{IU} / \mathrm{L})$ \\
\hline Mean titer $=262.40$ & 1 month after booster dose $(\mathrm{n}=30)$ \\
\hline
\end{tabular}

Anti-HBs response among the different groups of vaccinated individuals was in figure 2. Each group comprised 30 individuals.

Table-IV

Anti-HBs response among different socioeconomic condition groups

\begin{tabular}{lcccc}
\hline Class & \multicolumn{2}{c}{ Anti-HBs titer (IU/L) } & Total & Significance \\
& Positive $(>10)$ & Negative $(<10)$ & & \\
\hline High & $47(95.92 \%)$ & $2(04.08 \%)$ & $49(32.67 \%)$ & $\mathrm{P}<0.05$ \\
Middle & $72(92.31 \%)$ & $6(07.69 \%)$ & $78(52.00 \%)$ & \\
Low & $14(60.87 \%)$ & $9(39.13 \%)$ & $23(15.33 \%)$ & \\
Total & $133(88.67 \%)$ & $17(11.33 \%)$ & 150 & \\
\hline
\end{tabular}

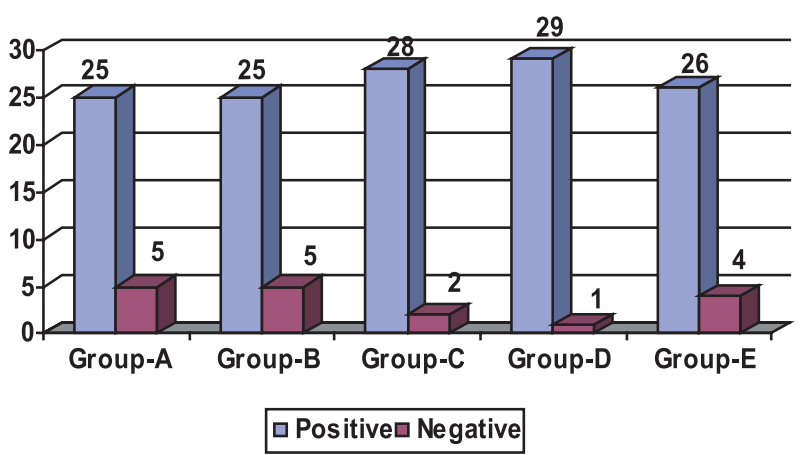

Fig.-2 : Anti-HBs response among the vaccinated individuals of different groups

\section{Discussion}

Debilitating and deadly, HBV infection has plagued human kind since the beginning of the recorded history. Though HBV infection is a significant health problem around the world, fortunately it is one of the oncoviruses that is vaccine preventable. HB vaccine induces anti-HBs response which can prevent $\mathrm{HBV}$ infection. Prevention of primary infection by vaccination is an important strategy to decrease the risk of chronic HBV infection and its subsequent complications. Studies have shown that childhood vaccination significantly reduced the rate of chronic $\mathrm{HBV}$ infection. ${ }^{20}$ 
The first generation hepatitis B vaccine, an inactive plasma derived vaccine became available in 1982. Subsequently the second generation $\mathrm{HB}$ vaccine, a DNA recombinant vaccine was also available for general use in 1986. Both of the vaccines are proven to be safe and efficacious. The efficacy of the recombinant vaccine is claimed to be around 95\% among children and $90 \%$ among adults. ${ }^{15}$ The effectiveness of vaccine has been demonstrated by observing the good humoral immune response in many countries since their implementation in EPI program and for other high risk groups. $^{21,22}$ Those studies were based on the development of anti-HBs titer, the protective limit of which is $>10 \mathrm{IU} / \mathrm{L}$, as accepted and recommended by WHO. ${ }^{23}$ Further, those studies were carried out to find out the relation of anti-HBs response to age, dose, body weight, obesity, genetic factor, past HBV infection, immunodeficiency disorder, immunosupression, cold chain factors etc. and how to improve overall immune status for successful elimination of HBV infection.

The immunization with $\mathrm{HB}$ vaccine has been in practice irregularly in our country for more than last 15 years and Bangladesh government has incorporated it in the EPI schedule since 2005 to cover all children. Now 3 doses of recombinant $\mathrm{HB}$ vaccine are given to every child along with DPT in the EPI program. The present study was designed to see the seroconversion status by measuring the anti-HBs titer among the individuals of different age and sex, vaccinated in both EPI and non-EPI schedule. A total of 190 individuals comprising 150 vaccinated and 40 non-vaccinated persons were enrolled in this study. Vaccinated 150 individuals were divided into five groups, designated as A, B, C, D and E with each group included 30 persons. While the non-vaccinated individuals served as control for this study. In order to see the immuno-protection offered by the vaccine and to detect good responders, hypo responders and non-responders to HB vaccine, serum anti-HBs titer was measured for individuals from group A, C and D where samples were taken within one to six months of completion of their vaccination schedule. On the other hand, serum samples from group B and $\mathrm{E}$ were tested after six months post vaccination to see the anti-HBs titer at different time interval for ensuring immuno-protection.

Out of 150 vaccinated persons, who received 3 doses or booster doses of Hepatitis B vaccine, 133 developed seroconversion with protective level of immunity in the current study. The overall sero-protectivity rate (anti-HBs titer $>10 \mathrm{IU} / \mathrm{L}$ ) among the vaccinated individuals was found to be $88.67 \%$, while all 40 non-vaccinated individuals who served as controls in the present study had anti-HBs titer below the protective level ( $<10$ IU/L) (Table-I). Two separate studies conducted in South Africa and Brazil reported the antibody response among the $\mathrm{HB}$ vaccinated individuals as 86.80\% and $90.00 \%$ respectively. ${ }^{24,} 25$ Further, a study conducted in Bangladesh among EPI-vaccinated children, the rate of sero-protectivity was found to be $92.20 \% .{ }^{26}$ So, as far as the seroconversion after HB vaccine is concerned, the results of the present study are well consistent with that of others.

In the present study, among 90 vaccinated individuals (sample collected within 1 to 6 months after completion of vaccination series), 61(67.78\%) were good responders, 21(23.33\%) were hypo-responders and 08(08.89\%) were nonresponders respectively (Table-II). Several studies have documented the percentage of non-responder between $11.9 \%$ and $21 \%$ among the vaccinees in the different parts of the world. ${ }^{27,} 28$ The percentage of non-responder in this study is well consistent with other studies.

Several studies have found serologic evidence of HBV protection for more than 10 years in $70 \%$ to $80 \%$ of those who were vaccinated. ${ }^{29}$ What will happen in future to these persons shown to have protective immunity now is an unresolved issue without subsequent follow-up. Thus only long term follow up for vaccinated persons can predict their ultimate immune status.

Mean anti-HBs titer was estimated for vaccinated individuals representing group $\mathrm{C}$ and $\mathrm{D}$ who received 3 doses and booster dose schedule of vaccination respectively. The mean titer of anti-HBs was found to be 262.40 IU/L among 3 doses recipients while it was $863.39 \mathrm{IU} / \mathrm{L}$ among booster dose recipients (Table-III). It revealed that mean titer is significantly higher in booster dose than in 3 doses recipients. Although literature suggests that protective immunity can be achieved by 3 doses of HB vaccine but booster dose definitely enhance immune response and it is recommended specially for persons labeled as non-responders after completion of their initial vaccination schedule.

Vaccinated individuals were categorized into low, middle and upper classes according to their monthly income based on criteria set by Bangladesh Bureau of Statistics. This study showed that people with low socio-economic conditions were found to have comparatively lower anti-HBs response $(\mathrm{P}<0.05)$ (Table-4). This may be correlated with malnutrition prevailing among individuals of low socio-economic class with consequent lower immunity. ${ }^{30}$

The present study recorded $11.33 \%$ vaccinated individuals having anti-HBs antibody titer below the protective level (Table-I). In fact, immune response depends upon a number 
of both host and immunogen factors. In this relation dose is an important factor affecting the anti-HBs response. Several studies have showed that the geometric mean titer induced by 10 and 20 microgram doses do not vary between plasma derived and recombinant hepatitis B vaccines. But 10 and 20 microgram doses of recombinant $B$ vaccine induce higher antibody titer than 2 and 5 microgram doses of the same. ${ }^{31}$ In this study, children and adult received a recommended dose of 10 and 20 microgram of recombinant $\mathrm{HB}$ vaccine respectively and the satisfactory rate of seroconversion can be correlated with the optimum dose.

Like optimum dose, route i.e. the site of inoculation is also very important for adequate immune response. Vaccine given to buttock or intradermally resulted in low antibody titer than intramuscular injection into deltoid region for the adults or outer aspect of thigh in case of children. ${ }^{32,33}$ In this study, adults were given $\mathrm{HB}$ vaccine intramuscularly in their deltoid muscle while children received intramuscularly at the outer aspect of the thigh. These correct sites of vaccinations can also be correlated with good immunity noted in the current study. Further, the effectiveness of vaccine also depends upon proper maintenance of 'cold chain'. Hepatitis B vaccine should be stored at 2 to 8 degree Celsius to maintain its potency. The vaccines that were received by people in this study were maintained in proper cold chain. So it is assumed that maintenance of proper cold chain in this study is also a contributing factor for satisfactory immune response among vaccinees. It is also mention worthy that, all relevant history and clinical observations were noted for each of the study cases into data sheet and these records also do not indicate any factor grossly associated that can adversely affect the immune response.

The protective immunity of $\mathrm{HB}$ vaccine is directly related to the development of anti-HBs titer with a minimum level of 10 IU/L is considered as protective level. ${ }^{3}$ On the basis of immune response (anti-HBs titer) to HB vaccine that develops within one to six months after completion of vaccine schedule, vaccinated individuals are categorized into goodresponder (titer>100 IU/L), hypo-responder (titer between 10-100 IU/L) and non-responder ( titer $<10 \mathrm{IU} / \mathrm{L}$ ). ${ }^{17}$ Both good and hypo responder are protected against HBV infection but non-responder remains susceptible to HBV infection. Non-responders to HB vaccine can be due to both host factors and vaccine itself. Factors affecting the efficacy of vaccine are more or less ruled out in this study, but host factors other than immunosuppression which has been ruled out can be genetic one that may affect the antibody response. Certain HLA types are found among the non-responders.
These are DR3, DR7, DR14 alleles especially when they are present on the extended haplotypes HLA B8-DR3-SCo1, HLA B44-DR7-FC31 and HLA Bw54-DR4-DQW4. ${ }^{34, ~ 35, ~} 36$ This genetic evaluation for non-responders was beyond the scope of this study.

It is already stated that, to label a vaccinated person as nonresponder, anti-HBs titer has to be determined within 1 to 6 months after completing the vaccination schedule. Out of total 17 persons found to have anti-HBs titer below protective level in this study, 9 individuals from group B and E had no post vaccination testing for labeling them as non-responders. So it is difficult to comment whether these persons were non-responder from the beginning of their vaccination or they responded initially but later on the anti-HBs titer waned off to protective level.

\section{Conclusion}

HBV is moderately endemic in countries like Bangladesh. Therefore immunization of infants and high risk groups is an important strategy to prevent $\mathrm{HBV}$ infection and to reduce the burden of chronic HBV infection. If standard vaccination practices are followed, most individuals will mount an antiHBs response which is sufficient to prevent HBV infection. ${ }^{37}$ The current study demonstrates that the over all seroconversion rate after 3 doses and booster doses of hepatitis B vaccination in both EPI and non-EPI schedule is efficacious up to $88.67 \%$. The $11.33 \%$ those who showed anti-HBs titer below the protective level in the present study may be due reasons incurred by both host factors and vaccine itself.

Persistence of protective immunity is a vital issue for any vaccine. Some persons vaccinated as infants against respiratory diseases that are more easily transmissible, such as measles, mumps, or pertusis, have been shown to lose protection from infection during adolescence, leading to recommendations for booster dose of these vaccines during adolescence. ${ }^{38,39}$ If the immunity engendered by hepatitis $B$ vaccine wanes in older adolescents, young adults and high risk groups, then $\mathrm{HBV}$ infection could occur in adolescents and adulthood. In fact, scientists engaged in research with $\mathrm{HB}$ vaccination and immunity is clearly in two schools of thought. One group strongly believes that, routine booster dose is unnecessary as immunologic memory is sufficient to prevent $\mathrm{HBV}$ infection among the vaccinee. While others believe that, it is better to give them a booster dose that will cause not only a boosting effect in previously responder but also may induce an immune response among a majority of non-responders shown in different parts of the world. 
Further, as far as the spacing between 3 doses of vaccine is concerned, the present recommendation is that there should be at least 2 months interval between the $2^{\text {nd }}$ and the $3^{\text {rd }}$ dose in order to achieve the adequate immune response..$^{40}$ Many countries are also practicing this recommended interval in their EPI schedule.

Finally, it is recommended that, all high risk groups and prospective mothers should receive $\mathrm{HB}$ vaccine in order to achieve the commendable success in $\mathrm{HB}$ vaccination for a moderately endemic country like Bangladesh.

\section{Conflict of interest: None}

\section{References}

1. WHO. Hepatitis B: World Health Organization Fact Sheet 204, 2000.

2. $\quad$ Lee WM. Hepatitis B infection. N Engl J Med 1997; 337: $1733-1745$.

3. Mahoney FJ. Update on diagnosis, management, and prevention of hepatitis B virus infection. Clin Microbiol Rev 1999; 12: 351 - 366.

4. Margolis HS, Alter MJ, Hadler SC. Hepatitis B: evolving epidemiology and implications for control. Semin Liver Dis 1991; 11: 84 - 92.

5. Alter M. Epidemiology of hepatitis B in Europe and worldwide. J Hepatol 2003; 39: S64 - S69.

6. Toukan A. Strategy for the control of hepatitis B virus infection in the Middle East and North Africa. Vaccine 1990; 8(suppl): S117 - S121.

7. McQuillan GM, Townsend TR, Fields HA, et al. Seroepidemiology of hepatitis B virus infection in the United States. Am J Med 1989; 87(suppl 3A): 5S - 10S.

8. Gibney L, Saquib N, Metzger J, et al. Human immunodeficiency virus hepatitis B C and D in Bangladesh trucking industry; prevalence and risk factors. Int $\mathrm{J}$ Epidemiol 2001; 30: 878 - 884.

9. De Francisco A, Hall AJ, Alam N, et al. Hepatitis B infection in Bangladeshi mothers and infants. Southeast Asian J Trop Med Public Health 1999; 30: 296 - 298.

10. Rahman R, Amanullah, Sattar H, et al. Sero-epidemiological study of hepatitis B virus infection in a village. Bangladesh Med Res Counc Bull 1997; 23(2): 38 - 41.

11. Rumi MA, Begum K, Hasan SM, et al. Detection of hepatitis $\mathrm{B}$ surface antigen in pregnant women attending a public hospital for delivery: implication for vaccination strategy in Bangladesh. Am J Trop Med Hyg 1998; 59(2): 318 -22.

12. Khan M, Zaki KMJ, Ahmed KU. Clinical profile: Prognostic index in hepatocellularcarcinoma. Bangladesh Med Res Coun Bull 1991; XVII: 49-62.
13. Bancroft WH, Snitbhan R, Scott RM, et al. Transmission of hepatitis $\mathrm{B}$ virus to gibbons by exposure to human saliva containing hepatitis B surface antigen. J Infect Dis 1977; 135: 79 - 85 .

14. Scott RM, Snitbhan R, Bancroft WH, et al. Experimental transmission of hepatitis B virus by semen and saliva. J Infect Dis 1980; 142: 67 - 71.

15. Bowes M. American Academy of Family Physicians. Pathways of controlling Hepatitis A and B: Vaccination, Prevention and Treatment. October, 2003.

16. Siebert D and Locarnini S. Hepatitis b: issues in laboratory diagnosis and vaccination. Aust Prescr 1998; 21: 72 - 5.

17. Zuckermann JN. Non-response to hepatitis B vaccines and the kinetics of anti-HBs production. J Med Virol 1996;50: $283-8$.

18. Zuckerman JN, Sabin C, Craig FM, et al. Immune response to a new hepatitis $\mathrm{B}$ vaccine in healthcare workers who had not responded to standard vaccine : randomised blind doseresponse study. Br Med J 1997; 314: 329 - 33.

19. Ronald W. Ellis,ed. Hepatitis B vaccine in clinical practice. New York: Marcel Dekker, 1993: 301.

20. Harpaz R, MacMohan BJ, Margolis HS. Elimination of new chronic hepatitis $B$ virus infection : results of the Alaska immunization program. J Infect Dis 2000; 181: 413 - 418.

21. Ni YH, Chang MH, Huang LM, et al. Hepatitis B virus infection in children and adolescence in a hyperendemic area: 15 years after mass hepatitis B vaccination. Ann Intern Med 2001; 796 - 800 .

22. Bonanni P, Pesavento G, Bechini A, et al. Impact of universal vaccination programmes on the epidemiology of hepatitis $B$ : 10 years of experience in Italy. Vaccine 2003; 21: 685 - 691.

23. West DJ, Calandra GB. Vaccine induced immunologic memory for hepatitis B surface antigen: implication for policy on booster vaccination. Vaccine 1996; 14(11): 1019 - 1027

24. Tsebes KV, Burnett RJ, Hlungwani NP, et al. The first five years of universal hepatitis B vaccination in South Africa; evidence for elimination of HBsAg carriage in under 5-yearchildren. Vaccine 2001; 19: 3919 - 3926.

25. Ribeiro TM and Azevedo RS. Seroconversion of hepatitis $B$ vaccine in infants related to the mothers serostatus in a community of Sao Jose dos Campos, state of Sao Paulo, Brazil. Clinics 2006; 61: 5.

26. Khan TM. Evaluation of immnue status against hepatitis B following Hep-B vaccination under EPI programme. 2006.

27. Craig B, Mitchell I, Anthony J, et al. Serological Hepatitis B Immunity in Vaccinated Health Care Workers. Arch Intern Med 1999; 159: 13. 
28. Zeeshan. Immune response after hepatitis B vaccine in different parts of the world. BMC Infect Dis 2007; 7: 120.

29. Malay S, Tizer K, Lutwick LL. Current update of pediatric hepatitis vaccine use. Pediatr Clin North Am 2000; 47: 395406.

30. Zaman K, Baqui AH, Yunus M, Sack RB, Chowdhury HR, Black RE. Malnutrition, cell mediated immune deficiency and acute upper respiratory tract infections in rural Bangladeshi children. Acta Paediatr 1997; 86(9): 923 - 7.

31. Troin F. Degos F, Brechot C, Courouce AM, Adamowicz P, Saliou P, Laplanche A, Benhamou JP, Giard M. Randomized doses : Range study of Recombinant hepatitis B vaccine Produced in Mammalian cells and Containing the S and pre S 2 sequences. J Infect Dis 1989; 160(2): 199 - 204

32. Ukene T, Esber H, Bessette R, Praks T, Crocker B. Site of injection and response to hepatitis B vaccine. N Eng J Med 1985; 313(9): 579 -580.

33. Sharifi-Mood B, Izadi S, Salehi M, Qaedi H. Comparison of Immunogenicity of Low dose ID Hepatitis B Vaccine with Standard dose IM vaccination in Young Healthy Iranian Adults. Journal of Hepatitis Monthly 2008; 8(2): 111 114.

34. Craven DE, Awdeh ZL, Kunches LM, Yunis EJ, Diestag JL, Werner BJ, Polk BF, snydman DR, Platt R, Crumpacker CS, Grady GF, Alper CA. Nonresponsiveness to hepatitis B vaccine in health care workers. Ann Inter Med 1986; 105: 356-360
35. Watanabe H, Matsuchita S, Kamikawaji N, Hirayama K, Okumura M, Sasazuki T. Immune suppression gene on HLA-Bw54-DR4-DRw53 haplotype controls nonresponsiveness in humans to hepatitis B surface antigen via CD8+ suppressor T cell. Hum Immunol 1988; 22: 9 17.

36. Zucman SC, Gimenez JJ, Wambergue F, Albouze BL, Naret C, Moynot A, Jungers P, Bach JF. Distict class II alleles determine antibody response to vaccination with hepatitis B surface antigen. Kidney Int 1998; 53: 1626 - 1630.

37. Atkintson WL, Pickering LK, Schwartz B et al.General recommendations on immunization. Recommendations of the advisory committee on immunization practices (ACIP) and the American Associations of Family Physicians (AAFP). MMWR Recomm Rep 2002 ;55: 1- 35.

38. Watson JC, Hadler SC, Dykewich CA, Reef S, Philips L. Measles, mumps and rubella: vaccine use and strategies for elimination of measles rubella and congenital rubella syndrom

39. Border KR, Cortese MM, Iskander JK, et al. Preventing tetanus, diphtheria, and pertussis among adolescents: use of tetanus toxoid, reduced diphtheria toxoid and acellular pertussis vaccines: recommendations of Advisory Committee on Immunization Practices. MMWR Recomm Rep 2006; 55: 1-34

40. CDC. Protection against viral hepatitis: recommendatios of the Immunization Practices Advisory Committee (ACIP). MMWR 1990; 39: 5 - 22. 